\section{A New Age for Recombinant Human Thyroid-Stimulating Hormone?}

TO THE EDITOR: I read with interest the recent article by Tuttle et al. (1). For years, patients have been prepared for ${ }^{131} \mathrm{I}$ administration after thyroid cancer surgery. It is believed that the increase of thyroid-stimulating hormone concentration by either thyroid hormone withdrawal (the traditional method of preparation) or injection of recombinant human thyroid-stimulating hormone (rhTSH) promotes ${ }^{131} \mathrm{I}$ uptake and retention in remaining radioiodine-avid cells. It is somehow also believed that the same patient preparation applies regardless of whether ${ }^{131} \mathrm{I}$ is given for diagnosis or for therapy. There is, however, no in vivo evidence that the thyroidal absorbed dose rate or dose per unit of administered therapeutic ${ }^{131} \mathrm{I}$ activity (or any other pivotal radiobiologic factor) has clear benefits over the traditional patient preparation. On the contrary, there are data in contrast to the common paradigms (2-6).

An $\mathrm{R} \times \mathrm{C}$ contingency table is a table with $\mathrm{R}$ rows and $\mathrm{C}$ columns. The $\chi^{2}$ test is used to analyze the relationship between 2 discrete variables, where one variable, depicted in the rows, has $\mathrm{R}$ categories and the other variable, depicted in the columns, has $\mathrm{C}$ categories. Consequently, the $P$ values given in the contingency tables of Tuttle et al. (1) are correct, but their use and interpretation for a single C category are not. For instance, although short-term recurrence rates were similar between the rhTSH and withdrawal groups $(P=$ not significant), the best clinical outcome (no clinical evidence of disease) was significantly higher in the rhTSH group than in the withdrawal group (the corresponding $P$ values are not the 0.10 and 0.021 reported in Tables 5 and 6 , respectively), regardless of the thyroglobulin cutoff value (1). Furthermore, it seems doubtful whether the outcome category "thyroid bed uptake only" should have been included in contingency tables containing a total of 394 patients, of whom only 291 (73.9\%) underwent follow-up diagnostic whole-body scanning (1). These issues should be the subject of a published erratum, as should "radioactive ablation of iodine remnants" on the sixth page of the article, and " $n=394$ " instead of the correct $n=371$ in Table 7 (1).

Most of the study limitations have already been discussed by the authors (1). In an effort to explain the significantly higher rate of no clinical evidence of disease in the rhTSH patients than in the withdrawal patients, I noted that the former received a "slightly" higher administered ${ }^{131} \mathrm{I}$ activity (in fact, it was not slightly but significantly higher, at $P=0.01)$, were significantly older $(P=$ $0.03)$, had a longer surgery-to-ablation interval, and underwent an essentially different preparation (1). Interestingly, the absorbed dose per unit of administered ${ }^{131}$ I activity in the thyroid remnant was found to correlate positively with age in our patients after thyroid hormone withdrawal.

I believe, in some contrast to the presented methodology and conclusions (1), that high success rates for ablation and low recurrence rates are achievable in totally or near-totally thyroidectomized patients without significantly compromising the patient's quality of life. These results can be achieved by a single administration of less than $2 \mathrm{GBq}$ of ${ }^{131} \mathrm{I}$ and by thyroid hormone

COPYRIGHT @ 2009 by the Society of Nuclear Medicine, Inc. supplementation started or restarted within 10-20 d after surgery or thyroid hormone withdrawal (2-6). Such an approach is particularly reasonable when the overall costs of rhTSH-aided therapy (in certain countries or certain patients) are much higher than those of thyroid hormone withdrawal. However, the optimal patient-specific timing and dosage for ${ }^{131} \mathrm{I}$ treatment of thyroid cancer have yet to be determined.

\section{REFERENCES}

1. Tuttle RM, Brokhin M, Omry G, et al. Recombinant human TSH-assisted radioactive iodine remnant ablation achieves short-term clinical recurrence rates similar to those of traditional thyroid hormone withdrawal. J Nucl Med. 2008;49:764-770.

2. Medvedec M, Grosev D, Pavlinovic Z, Dodig D. Preparation of thyroid cancer patients for scintigraphy and therapy with radioiodine [abstract]. Eur J Nucl Med. 1999;26:1088.

3. Behr TM, Behe MP, Gratz S, Huefner M, Becker WH. Factors influencing the dosimetry and the therapeutic outcome of ${ }^{131} \mathrm{I}$ therapy of differentiated thyroid cancer (DTC) [abstract]. J Nucl Med. 2001;42(suppl):242P.

4. Grigsby PW, Siegel BA, Bekker S, Clutter WE, Moley JF. Preparation of patients with thyroid cancer for ${ }^{131}$ I scintigraphy or therapy by 1-3 weeks of thyroxine discontinuation. J Nucl Med. 2004;45:567-570.

5. Medvedec M, Dodig D. Has come the day to do away with thyroid remnant ablation targeting 300 gray (Gy)? [abstract]. J Nucl Med. 2007;48(suppl 2):16P.

6. Medvedec M, Dodig D. High ablation success rate achieved by the lowest I-131 activity to date [abstract]. J Nucl Med. 2008;49 (suppl 2):8P.

\section{Mario Medvedec Clinical Hospital Centre Zagreb Zagreb, Croatia}

DOI: 10.2967/jnumed.108.058255

REPLY: We thank the reader for the constructive criticism of our recent article describing similar short-term clinical outcomes using either recombinant human thyroid-stimulating hormone (rhTSH) or traditional thyroid hormone withdrawal preparation for radioactive iodine remnant ablation (1). Like the reader, we agree that a short period of thyroid hormone withdrawal followed by moderate administered activities of radioactive iodine is successful in achieving high rates of remnant ablation (in fact, in our study this was the gold standard to which patients prepared with rhTSH were compared). Furthermore, by minimizing the period of thyroid hormone withdrawal, the symptoms of hypothyroid withdrawal (although still present) are certainly less clinically significant than are prolonged periods of thyroid hormone withdrawal before therapy.

Rather than concluding that rhTSH preparation was superior to thyroid hormone withdrawal based on nonrandomized, retrospective data with short-term follow-up, we were careful to conclude that rhTSH-assisted ablation is simply a "safe and effective option" to thyroid hormone withdrawal. We continued by stating that the "choice of preparation for [radioactive iodine remnant ablation] ([thyroid hormone withdrawal] vs. rhTSH) should be made on the basis of patient's quality of life and the 'cost' of hypothyroidism versus the cost of rhTSH" (1).

As with any retrospective study there are significant limitations, which we carefully pointed out in the discussion. Even though the differences in age $(44 \pm 15 \mathrm{y}$ for thyroid hormone withdrawal vs. $48 \pm 15$ y for rhTSH, $P=0.03)$ and administered activity $(3,811-$ $\mathrm{MBq}$ [103-mCi] median for thyroid hormone withdrawal vs. 